\title{
Creencias ambientales mexicanas. La importancia de construir sociedades resilientes
}

\author{
Orlando Puente Zubiaur
}

\begin{abstract}
Resumen
¿Los animales y las plantas tienen tanto derecho a existir como los humanos? En dónde usted vive, ¿cómo diría que es la situación del medio ambiente? ¿Se había preguntado alguna vez la opinión de los mexicanos hacia éste? La dimensión ambiental en los albores del sig/o xxl es un libro basado en la opinión pública, y los datos que ahí se presentan fueron obtenidos de encuestas nacionales sobre el dominio del tema del medio ambiente en México. La gran mayoría de las preguntas de la encuesta nacional impactan en el lector de manera personal, lo que obliga a la reflexión consciente de los problemas que enfrenta el mundo, nuestro país, nosotros mismos y las siguientes generaciones. Finalmente, el libro concluye al enfatizar la importancia de la educación y la difusión de los problemas ambientales para la construcción de sociedades más resilientes.
\end{abstract}

Palabras clave: medio ambiente, dicotomía naturaleza/cultura, creencias, educación ambiental.

\section{MEXICAN ENVIRONMENTAL BELIEFS. THE IMPORTANCE OF BUILDING RESILIENT CITIES}

\begin{abstract}
Do animals and plants have as much right to exist as humans do? How would you say is the environment situation where you live? Have you ever wondered the opinion of Mexicans towards the environment? La dimensión ambiental en los albores del sig/o xxl is a book based on public opinion, where the data presented was obtained from national surveys on the domain of the environment topic in Mexico. The vast majority of the questions in the National Survey impact the reader in a personal way, forcing a conscious reflection of the problems faced by the world, our country, ourselves and the following generations. Finally, the book concludes by emphasizing the importance of education and the diffusion of environmental problems for the construction of more resilient societies.
\end{abstract}

Keywords: environment, dichotomy nature/culture, beliefs, environmental education.

Recepción: 03/10/2018. Aprobación: 26/08/2020. Dol: http://doi.org/10.22201/cuaieed.16076079e.2020.21.6.14 
Egresado de la licenciatura en Historia del Instituto Cultural Helénico y de la Maestría en Estudios Históricos de la Universidad Autónoma de Querétaro. Actualmente está adscrito al Doctorado en Estudios Interdisciplinarios sobre Pensamiento, Cultura y Sociedad, de la Universidad Autónoma de Querétaro. Fue investigador en el Museo de Historia de Celaya, siendo responsable de la investigación de exposiciones permanentes y temporales, capacitación en difusión histórica, producción de radio cultural y miembro del equipo de conservación de cuerpos áridos del Museo de Momias de Celaya. Desde 2011 es profesor de historia del Arte, Museología e Historia de la Arquitectura en diversas universidades privadas en Celaya, Guanajuato. Sus intereses de investigación son la Historia del Arte, Historia Cultural e Historia Ambiental.

\section{Introducción}

La dimensión ambiental en los albores del sig/o xxi ha sido de mi agrado para ser referenciada en mi investigación doctoral, principalmente porque aborda la temática sobre las creencias, los hábitos y la memoria socioambiental. En mi trabajo de investigación, he pretendido construir una encuesta que me ayude a observar las actitudes ambientales de una población. Por lo anterior, me fue útil aplicar algunas de las interrogantes propuestas en este libro, que llenó todas mis expectativas de lo que uno mismo y la población en su mayoría respondería, así como del significado ambiental que esto conlleva. De igual manera, es interesante puesto que acerca al lector a comprender el nivel de impacto que los problemas ambientales ocasionan en las sociedades humanas, en particular, como en el planeta tierra, en general.

\section{La construcción del libro}

La dimensión ambiental en los albores del sig/o xxi forma parte de una colección de 26 obras titulada Los mexicanos vistos por sí mismos. Los grandes temas nacionales. Ésta fue el resultado analítico de diversas encuestas nacionales realizadas por el Área de Investigación Aplicada y Opinión del Instituto de Investigaciones Jurídicas de la Universidad Nacional Autónoma de México (UnAm). Fueron 93 investigadores y 48 jóvenes asistentes dirigidos por Julia Isabel Flores Dávila, quienes se encargaron de diseñar y levantar 25 encuestas - a 1200 casos cada una-, en todo el país.

La idea surgió en 2014 — aunque sigue siendo una obra muy actualizada-, en medio de un contexto de profunda incertidumbre e indignación por la desaparición forzada de los 43 normalistas de Ayotzinapa. ¿¿Cuál es el papel de la unAm en estas realidades?" se preguntaba el entonces rector de la Universidad, José Narro Robles: 
fotografía: es una imagen de un momento y un lugar determinado. De la percepción que los individuos tienen de sí mismos, porque "pone en juego el entramado de conocimientos y sentimientos contenidos en las respuestas" (p. 22). Las respuestas siempre están influidas por el contexto y, en este caso, la encuesta fue realizada en noviembre de 2014, unos meses después del problema de los estudiantes de Ayotzinapa, justo cuando la opinión y la atención pública se centraban concretamente en eso. Es claro que esto tuvo ciertas implicaciones en las respuestas de la gente que, según refieren los autores, consideraron los problemas ambientales en séptimo lugar de una lista de diez opciones (p. 42). A pesar de lo anterior, las encuestas sirven de contraste entre la teoría y la práctica; parafraseando a Ricardo Pozas en el Preámbulo, "entre el saber académico respecto de los problemas estudiados científicamente" (p. 26).

\section{Contrastando datos de la realidad ambiental}

¿Considera usted que el cambio climático ha afectado su vida diaria? ¿Cuánto cree usted que sabe sobre las soluciones de los problemas ambientales? ¿Se había hecho alguna vez este tipo de preguntas? Esta obra se basa en el supuesto de que las percepciones ambientales son construcciones sociales, definidas como la manera en la que cada individuo aprecia y valora su entorno, natural y social. Son dinámicas e históricas. Se modifican constantemente con base en nuevas informaciones e interacciones sociales que ocurren en una comunidad:

Las percepciones, junto con los valores y el conocimiento, conforman las actitudes ambientales, es decir la manera en la que las personas construyen su relación con el entorno, interpretan los cambios que ocurren en él, explican sus causas y proponen soluciones (p. 31).

En este sentido, es interesante el planteamiento inicial de los autores. Ellos sugieren que para los encuestados los problemas ambientales serios son los que pueden ubicarse claramente en su entorno inmediato, como la contaminación del aire o del agua. No obstante, otros problemas no tan visibles. Como el cambio climático, suelen observarse tan alejados de la cotidianidad, que difícilmente se perciben. Los autores explican que la encuesta refleja preocupación de la población hacia el cambio climático, pero más de la mitad no tiene una idea correcta de lo que es (p. 135).

El análisis que los autores realizaron de los datos recabados se entrecruza con diversas teorías sobre el daño ambiental y la relación de éste con las percepciones humanas. Como sugiere Jared Diamond (2006, cap. 14), el deterioro ambiental es tan gradual que muchas veces la gente es incapaz de observar los cambios y discernir su magnitud acumulada. A esto le llama amnesia del paisaje: el cambio tan paulatino del medioambiente pasa inadvertido para quienes, por haber estado envueltos por ese paisaje año tras año, lo observan inamovible. De igual forma, William Hirst y Charles B. Stone (2015, p. 106-107) proponen que un estudio más completo sobre la memoria colectiva debe contemplar también el olvido colectivo. Lo que una sociedad construye de su pasado en parte estará 
relacionado con lo que olvida. Conjugando las posturas de Diamond y Hirst/ Stone, resultan evidentes los análisis sobre el cambio climático en el trabajo de Ímaz. Ocurre de forma tan paulatina y la memoria de la gente — se sugiere en La dimensión ambiental_ "es muy corta y expresiones como 'nunca había hecho tanto calor', 'nunca había llovido tanto', 'este frío no se había vivido jamás' son constantes en las conversaciones año con año" (p. 139).

Los autores, en esta obra, describen la responsabilidad que los gobiernos e instituciones tienen sobre los problemas ambientales. Por ejemplo: la crítica a la falta de presupuesto, al interés en el cuidado del medio ambiente, a las políticas de protección, la educación ambiental y la difusión de los problemas, son temáticas constantes que van hilando el discurso del libro. Desde el capítulo 2, "El México que nos rodea", hasta el 7 "Saberes y opiniones", los autores evidencian la falta de interés y/o conocimiento que la autoridad empeña en dichos problemas. También dedican muchas palabras a explicar la importancia de políticas públicas adecuadas en el manejo de los recursos y ofrecen cifras oficiales muy interesantes. Por ejemplo, explican que el costo total del agotamiento y degradación ambiental de México asciende a 5.7\% del producto interno bruto, en contraste con 1.2\% (p. 40) que se dedica a la protección ambiental a través de políticas públicas, campañas educativas, aplicación de energías renovables, etcétera.

En promedio, los 34 países de la OCDE, recaudan 1.59\% de su PIB mediante impuestos de este tipo. México, por lo contrario, al ofrecer un subsidio a la producción y consumo de combustibles fósiles es el único país que "subsidia" procesos contaminantes por un monto que equivale a 1.28\% de su PIB (p. 40).

Paradójicamente, sugieren los autores, el gobierno destina recursos que aumentan la degradación del medio ambiente. Sin embargo, quizás la postura más recurrente en el libro tiene que ver con la educación y el manejo de la información. Muchas de las preguntas de la Encuesta Nacional evidencian la falta de conocimiento y comprensión de la población acerca de los problemas de este tipo. Esto pasa no sólo con el cambio climático —como ya se mencionó en líneas anteriores-, sino también con tecnologías alternativas, como los paneles fotovoltaicos, calentadores solares, recuperación de lluvia y tecnologías ahorradoras de agua. Según los datos recabados en la encuesta, más de 85\% mencionaron no utilizar ninguna de las anteriores, ya que no sabían que existían o no contaban con los recursos para instalarlos en sus hogares (p. 108). Los autores sugieren que el ingreso familiar es un factor determinante; aunque la falta de información adecuada en las campañas de gobierno y la falta de interés de los medios de comunicación, acrecientan el problema. Explican que la ausencia de la temática ambiental en la televisión coincide con la poca importancia que le otorgan los encuestados: "siendo probablemente un factor clave, junto con la educación, en la conformación - o la no conformación- de una cultura ambiental" (p. 154).

Evidentemente, la perspectiva general del libro no quedaría cubierta si no abordamos la participación y responsabilidad que los autores atribuyen 
a los encuestados. Ciertamente, como ellos mencionan, la participación y el involucramiento de la población tienen estrecha relación con una gobernanza ambiental más adecuada. $Y$, en este sentido, los autores se muestran esperanzados por la disposición personal interpretada en la encuesta. A la gran mayoría (71\%) le preocupaban los problemas ambientales —algunos por ser conscientes de la relación entre la degradación ambiental y la calidad de vida, en otros casos debido al bombardeo mediático que ha posicionado este tema en el cine, la televisión y la prensa-. Sin embargo, principalmente quienes tienen estudios de licenciatura o posgrado se mostraban con más interés de participar en organismos y colectivos de protección (p. 80-82). La gente parecía dispuesta a pagar más por los productos en pro del medio ambiente. Además, consideraban que uno de los grandes problemas estaba relacionado con los bajos impuestos y la ineficiencia en la recaudación de los mismos. El capítulo 4 "Del dicho al hecho" habla enteramente de este esperanzador escenario. Excepto por un punto importante, cerca del 89\% de los encuestados afirmaron nunca haber participado en alguna asociación o firmado alguna petición ambientalista. Como bien dice el refrán: "del dicho al hecho, hay mucho trecho".

Los autores ponen "el dedo en la llaga", al señalar la contradicción existente entre las percepciones ambientales y las actitudes. Sugieren que las actitudes ambientales (lo que se hace, lo que ocurre) están ampliamente delimitadas por diversos factores como: el ingreso, las experiencias individuales, culturales y sociales. "De ahí que los resultados de las actitudes de los encuestados pueda distanciarse de sus percepciones e intenciones, en tanto unas y otras no tienen una relación lineal o directa" (p. 87). Es decir, los encuestados afirmaban estar interesados en mejorar las condiciones ambientales de su entorno, pero muy pocos de ellos habían participado activamente en acciones, campañas o, incluso, firmando peticiones colectivas (p. 88). En este sentido, los autores afirman que no fue posible analizar los elementos de forma aislada, por lo tanto, se basaron en la interpretación del conjunto de datos ofrecidos por la encuesta en general.

No obstante, quizás en este sentido los autores pecaron un poco de ingenuidad esperanzadora. Es posible que en gran medida tenga relación con un aspecto vital abordado en la misma presentación y que tiene mucho que ver con algunas de las creencias generalizadas, específicamente la dicotomía naturaleza-cultura. Históricamente, en Occidente se ha creído en una supuesta separación entre lo natural y lo humano. Herencia de la antigüedad, la dicotomía está presente incluso en el Génesis del Antiguo Testamento, desde el momento en que Dios creó a los humanos, a su imagen, para señorear al resto de los animales. En esta concepción del mundo, más allá de las ciudades se encuentra lo natural; como un oasis donde existe todo lo ajeno a lo humano, las ciudades han sido concebidas para resguardar a los humanos de un mundo "salvaje y hostil". Dicha dicotomía ha guiado las creencias occidentales como hábitos de acción y delimitado la interacción entre la humanidad y la naturaleza, considerada lo otro (Myllyntaus y Saikku, 2001, p. 143). Esta dicotomía está presente en múltiples creencias occidentales comunes, como las ideas generalizadas de una separación entre razón e instinto, humanos y animales, hábitat humano y naturaleza, entre 
muchos otros ejemplos. El antropocentrismo ha impuesto gradualmente la idea de la humanidad como poseedora, ordenadora y protectora del entorno natural y sus recursos (Milesi, 2013, p. 6).

Debido a esta dicotomía, la gente tiene muchas dificultades para comprender la relación intrínseca existente entre sociedad y naturaleza, que conforma el sistema socioambiental. Sin embargo, en las encuestas la gente suele responder lo "socialmente deseable", aunque en la práctica, como se demuestra en la misma encuesta, realmente no preste mucha importancia a los problemas ambientales, tal cual se pudo percibir cuando los encuestados colocaron los problemas ambientales en séptimo lugar de una lista de diez opciones. Aun así, vale la pena dar el voto de confianza, como sugieren los autores, e interpretar los resultados de la encuesta con resquicios de optimismo.

\section{A manera de conclusión}

Es momento de que cada uno de nosotros realicemos, como introspección, una reflexión en torno al medio ambiente. Los autores de este libro describen, de manera simplificada y muy amena, un discurso ambiental y una serie de preguntas resueltas por medio de encuestas, que impactan en el lector de manera personal, obligando a la reflexión y concientización de los problemas que enfrenta el mundo, nuestro país, nosotros mismos y el futuro de las siguientes generaciones.

También, La dimensión ambiental en los albores del sig/o xxI es una herramienta práctica y extremadamente útil para los estudios ambientales en general. Al no existir muchas investigaciones de este tipo en México, la obra carga con una doble responsabilidad: por un lado, abrir la mesa de discusión para valorar y revalorar los problemas ambientales, sus causas y el impacto de las creencias y perspectivas de la población. Esto es importante porque las voces de los mexicanos representan el pensamiento de la población, lo que permite observar las creencias y, por ende, los hábitos que rigen el día a día del mexicano. Sólo conociendo estos aspectos es posible redirigir la educación y las políticas en favor de sociedades más resilientes con el medio ambiente. Por otro, esta investigación aporta a la construcción de una ciudadanía informada, crítica y participativa, que impulse nuevas actitudes. Tal cual lo sugieren los autores (p. 168): "También es urgente socializar el conocimiento de las causas y los efectos de los procesos que afectan los socioecosistemas y que las personas tengan herramientas necesarias para tomar decisiones informadas". En las últimas décadas, la crisis ambiental ha cuestionado la sustentabilidad de la forma de vida humana, no la del planeta que encontrará la forma natural de adaptarse. Este tipo de investigaciones nos ayudan a comprender que la sustentabilidad va más allá de la política y la economía, nos compete a todos como sociedad. Como sugiere enrique Leff: "Pues junto con la crisis ambiental que pone en riesgo la vida, hoy vivimos una crisis moral que cuestiona el sentido de la vida humana. Y esto invita no sólo a una reflexión, sino a una re-educación..." (2009, p. 14) 


\section{Referencias}

* Diamond, J. (2006). Colapso: por qué unas sociedades perduran y otras desaparecen (Ricardo García Perez, Trad.). Debate.

* Hirst, W. y Stone, C. (2015). A Unified Approach to Collective Memory: Sociology, Psychology and the Extended Mind. En S. Kattago (Ed.), The Ashgate Research Companion to Memory Studies (pp. 103-115). Tallinn University.

- Leff, E. (2009). La esperanza de un futuro sustentable. Transatlántica de educación, (9). 94-103.

- Lezama, J. L. y Graizborg, B. (Coords.) (2010). Los grandes problemas de México. El Colegio de México. (Iv. Medio Ambiente).

* Milesi, A. (2013). Naturaleza y cultura: una dicotomía de límites difusos. De prácticas y discursos. Cuaderno de ciencias sociales, (2). http://biblioteca.clacso.edu. ar/Argentina/ces-unne/20141001053559/Milesi.pdf

- Mireya Atzala, I. G. (Coord.) (2015). La dimensión ambiental en los albores del sig/o XXI. Miradas desde la diversidad. Encuesta Nacional de Medio Ambiente. Universidad Nacional Autónoma de México.

Myllyntaus, T. y Saikku, M. (Coords.) (2001). Encountering the past in nature. Ohio University Press.

- semarnat. (2019). Informe de la Situación del Medio Ambiente en México, edición 2018. SEMARNAT.

\section{Cómo CITAR ESTE ARTículo}

* Puente Zublaur, Orlando. (2020, noviembre-diciembre). Creencias ambientales mexicanas. La importancia de construir sociedades resilientes. Revista Digital Universitaria (RDU), 21(6). Dol: http://doi.org/10.22201/ cuaieed.16076079e.2020.21.6.14 